\title{
Research on Intelligent Recommendation Business Model of Tourism Enterprise Value Platform from the Perspective of Value Cocreation
}

\author{
Qiongying Wang and Daijian Tang (D) \\ School of Tourism and Urban-Rural Planning, ZheJiang Gongshang University, Hangzhou 310018, China \\ Correspondence should be addressed to Daijian Tang; luyou@mail.zjgsu.edu.cn
}

Received 28 June 2021; Accepted 29 July 2021; Published 6 August 2021

Academic Editor: Shaohui Wang

Copyright (c) 2021 Qiongying Wang and Daijian Tang. This is an open access article distributed under the Creative Commons Attribution License, which permits unrestricted use, distribution, and reproduction in any medium, provided the original work is properly cited.

\begin{abstract}
With the rapid development of China's economy, people pay attention to their own quality of life, and tourism has become the first choice for people from all walks of life to relax themselves. Tourism travel has mainly developed from the form of travel agency registration to the form of online registration based on the network platform business model. Considering the value cocreation and the diversity of tourism enterprise platform, this paper puts forward the business model research of intelligent recommendation of tourism enterprise platform from the perspective of value cocreation. Firstly, the commonly used recommendation algorithms are introduced, which are collaborative filtering recommendation algorithm, content filtering recommendation algorithm, and association rule recommendation algorithm. Secondly, it analyzes the number of tourists and economic benefits of the business platform of tourism enterprises from April 2020 to April 2021 and also analyzes the business models of five modules under the tourism platform on different platforms. Finally, three recommendation algorithms are used to compare the comprehensive performance of five modules in different business models. Finally, we find that the rate of accuracy and recall of business is above $88 \%$, which can have good economic benefits and provide customers with high-quality recommendation service and good satisfaction.
\end{abstract}

\section{Introduction}

This paper discusses the intelligentization of tourism recommendation system based on WebGIS and gives the organization mode of urban tourism recommendation knowledge base based on WebGIS and the design scheme of inference engine in literature [1] using JSP and MapXtreme to realize the city tourism recommendation system. This scheme is very suitable for establishing intelligent city tourism information system. Applying data mining technology [2] to provide services for users' personalized needs is a hot spot in current tourism research. This paper introduces a tourism service intelligent recommendation system based on Apriori-MD algorithm, which is twice as efficient as Apriori algorithm. It has a wide application prospect in the tourism market. Because of the large error of data calculation in traditional recommendation system, literature [3] proposes an intelligent recommendation system for tourist attractions based on collaborative filtering. The overall structure diagram of the system is designed. The results show that the intelligent recommendation system has small data calculation error and good recommendation effect. Literature [4] deals with big data and its value-added tourism, which provides a better understanding of customer behaviors and preferences for making decisions. The rapid spread of new technologies in Morocco's tourism industry has created new demands and challenges, predicting the behaviour of tourists and providing personalized services. The new big data system helps tourism stakeholders make appropriate strategic decisions and promotes the development of tourism in Morocco. Literature [5] proposes an ontology-based CBR system for intelligent recommendation 
of tourism products and proposes an ontology-based case representation method and corresponding case retrieval method. It is proved that the system is effective. The characteristics of tourism show its importance to economic growth and the influence of information technology system. The TAST model proposed in literature [6] can effectively collect the personalized characteristics of tourism information. After generating the TAST model, the cocktail method is used to generate the recommended list of tourism packages, and the cocktail method is more effective in recommending tourism packages. The Internet has become the media of choice for many travelers to access travel information, as well as in Turkey. Literature [7] provides a new method for the marketing strategy of Turkish travel agencies. Using case-based reasoning algorithm, an intelligent system is established as a recommendation tool for travel planning. In mobile travel applications, active recommendation is particularly practical. Literature [8] proposes a method and research prototype based on smart space and active recommendation system technology, and the system implementing the proposed method helps tourists use their mobile devices. Literature [9] proposes the overall structure and functionality of an intelligent recommendation system that enables a user to identify his/her own destination, bundle a set of products, and make a travel plan for personalized travel. Literature [10] proposes an intelligent context-aware recommendation system, which makes intelligent reasoning to determine the weight or importance of different types of environment and time context. Tour guide applications rely heavily on location, resulting in inappropriate advice, which can be mitigated if proper personalization and content filtering are performed. Travel reviews and travel notes are two major ways to share social travel. More and more people are willing to share their travel experiences and feelings on the web, forming fragmented travel knowledge. Literature [11] proposes a multisource social media data fusion method, Crowd Travel, which provides users with travel information through crowd intelligent mining. Data mining, web mining, and knowledge management can be used to customize individual or group information services to improve tourists' satisfaction. For the concept of intelligent tourism electronic portal, literature [12] put forward the basic framework of intelligent tourism electronic portal.

\section{Recommendation Algorithm Analysis}

2.1. Collaborative Filtering Recommendation Algorithm. The core part of collaborative filtering recommendation algorithm is the selection of nearest neighbors [13]. The effectiveness and efficiency of this algorithm depend on the effectiveness and efficiency of neighbor user similarity calculation. There are three common calculation methods: cosine similarity, modified cosine similarity, and Pearson correlation coefficient, which will be introduced below.

The basic steps of collaborative filtering recommendation algorithm are as follows.
Input: current user ID, user-item scoring matrix UR, number of nearest neighbors $K$, maximum recommended value top- $N$;

Output: Recommended item list $L$;

Step 1: in the recommendation system based on user $\mathrm{CF}$, input the scoring data of all users for all items, and construct a matrix $R$ of $M * N$ as shown in Table 1 ( $M$ users and $N$ items), and $R_{i j}$ represents the scoring value of the $i$ th user for the $j$ th item. The rating of a user is considered as a vector in the $n$-dimensional term space. If the $i$ user does not rate the $j$ term, the matrix is expressed as $R_{i j}=0$.

In the recommendation in Table 1, the user and project scoring matrix reflects the recommendation relationship. If the score of the matrix is high, it means that the user has a high recommendation for this product and its commercial value is obvious. If there is a similar product recommendation value between different users, the product recommendation between different users will be compared, and different products will be recommended between different customers, thus increasing income.

Step 2: calculate the nearest neighbor; that is, calculate the user similarity value between the current user and all $M$ users, and select $K$ users with the largest user similarity as the nearest neighbors of the current user. As mentioned earlier, there are multiple methods to calculate user similarity, and the commonly used ones are the following.

2.1.1. Cosine-based similarity. Assuming that the set of items that users $i$ and $j$ have commented on together is represented by $i$, the similarity between the two users, $\operatorname{sim}(i, j)$, is defined as

$$
\operatorname{sim}(i, j)=\frac{\sum_{c \in \mathrm{I}} R_{i, c} R_{j, c}}{\sqrt{\sum_{c \in \mathrm{I}} R_{i, c}^{2} \sqrt{\sum_{c \in \mathrm{I}} R_{j, c}^{2}}}} .
$$

Among them, $R_{i, c}$ and $R_{j, c}$ represent the scores of users $i$ and $j$ on item $C$, respectively.

2.1.2. Modified cosine similarity. The modified cosine similarity calculation method subtracts the user's average score for all items from the user's score for a certain item, which avoids the calculation method of formula (1) not considering the difference between the average user scores. For example, some users tend to score high scores and low scores, thereby solving the problem of scoring scale among different users, and the calculation formula is defined.

$$
\operatorname{sim}(i, j)=\frac{\sum_{c \in \mathrm{I}_{i j}}\left(R_{i, c}-R_{i}\right)\left(R_{j f}-R_{j}\right)}{\sqrt{\sum_{c \in \mathrm{I}_{i}}\left(R_{i, c}-R_{i}\right)^{2}} \sqrt{\sum_{c \in \mathrm{I}_{j}}\left(R_{i f}-R_{j}\right)^{2}}} .
$$


TABLE 1: User-item scoring matrix.

\begin{tabular}{lccccc}
\hline User/item & $I_{1}$ & $I_{2}$ & $I_{3}$ & $\ldots$ & $I_{n}$ \\
\hline$U_{1}$ & $R_{11}$ & $R_{12}$ & $R_{13}$ & $\ldots$ & $\mathrm{R}_{1 n}$ \\
$U_{2}$ & $R_{21}$ & $R_{22}$ & $R_{23}$ & $\ldots$ & $\mathrm{R}_{2 n}$ \\
$\ldots$ & $\ldots$ & $\ldots$ & $\ldots$ & $\ldots$ & $\ldots$ \\
$U_{m}$ & $R_{m 1}$ & $R_{m 2}$ & $R_{m 3}$ & $\ldots$ & $R_{m n}$ \\
\hline
\end{tabular}

Among them, $R_{i}$ and $R_{j}$ represent the average scores of users $i$ and $j$ on the project, respectively.

\subsubsection{Person correlation coefficient}

For similarity between users $i, j, \operatorname{sim}(i, j)$ is also measured by Pearson correlation coefficient as follows:

$$
\operatorname{sim}(i, j)=\frac{\sum_{c \in \mathrm{I}_{i j}}\left(R_{i, c}-\bar{R}_{i}\right)\left(R_{j, c}-\bar{R}_{j}\right)}{\sqrt{\sum_{c \in \mathrm{I}_{i j}}\left(R_{i, c}-\bar{R}_{i}\right)^{2}} \sqrt{\sum_{c \in \mathrm{I}_{j}}\left(R_{j, c}-\bar{R}_{j}\right)^{2}}} .
$$

Step 3: $K$ nearest neighbors of the current user are obtained through the above methods, and the corresponding recommendation results are generated in the next step. If the nearest neighbor set of user $u$ is represented by $N$, the prediction score $P_{u, i}$ of user $u$ for item $i$ is calculated as follows:

$$
P_{u, i}=R_{u}+\frac{\sum_{n \in N} \operatorname{sim}(u, n) \times\left(R_{n, i}-R_{n}\right)}{\sum_{n \in N}(|\operatorname{sim}(u, n)|)},
$$

where $\operatorname{sim}_{n u}$ denotes the similarity between users $u$ and $n, R$ denotes the score of user $n$ on item $i$, and $R_{u}$ and $R_{n}$ denote the average score of users $u$ and $n$ on item $i$, respectively.

Step 4: through the above method, the current user can calculate the prediction scores of all commodities without interactive behaviors and finally select the list $L$ of top- $N$ recommended items with the highest prediction scores to the current user.

2.2. Content Filtering Recommendation Algorithm. In the tourism platform resource representation model and user consumption preference model, it has been proposed that, due to the variety of commodity types and different commodity characteristics and attributes involved in e-commerce, all categories of commodities are represented as a set $\left\{R_{i}\right\}$. It is a class $t$ commodity, which is represented by binary groups $\left\langle d_{i}, g_{i}\right\rangle$, where $d_{i}$ denotes the $i$ th attribute of the $t$ th commodity and $g_{i}$ denotes the set of values of the attribute $d_{i}$. The user's preferences on all categories of goods are expressed as a set $\left\{U_{t}\right\}$, where $U_{t}$ is the user's preferences on category $t$ goods. $U_{t}$ is represented by the triple $\left\langle a_{i}, v_{i}, w_{i}\right\rangle$, $i=1, \ldots, m$, where $a_{i}$ represents the $i$ th attribute of the $t$ th commodity. If the value of the attribute $a_{i}$ is numeric, $v_{i}$ denotes the value preferred by the user on the attribute; if the value of the attribute $a_{i}$ is nonnumeric, $v_{i}$ denotes the set of attribute values preferred by the user on the attribute; $W_{i}$ represents the user's weight on the attribute $a_{i}$, which is used to describe the degree to which the user pays attention to the attribute, and satisfies that $\sum_{i=1}^{m} w_{i}=1$ and $m$ is the number of $T$-type commodity attributes.

2.2.1. Mining Algorithm of Consumer Preference. Input is user's consumption preference on category $t$ goods $<a_{i}, v_{i}$, $w_{i}>$.

(i) Step 1: data preprocessing: extract the historical consumption records of users on the T-type goods, and sort them according to the time sequence of users' consumption. For each attribute $a_{i}$ of the $t$ type goods, the value set $v_{i}=\left\{v_{i}^{1}, v_{i}^{2}, \ldots, v_{i}^{n}\right\}$ is obtained, and $n$ is the number of times the user consumes the $t$-type goods. For each attribute $a_{i}$, execute step 2 through step 4 , and then go to step 5 .

(ii) Step 2: attribute value distribution probability calculation: if the value of $a_{i}$ is continuous, it is discretized by interval partition, and the partitioned interval is represented by $b_{k}$, and $v_{i}^{1}, v_{i}^{2}, \ldots, v_{i}^{n}$ is mapped to each interval. If $a_{i}$ is a discrete value, its value is expressed by $b_{k}$. The probability that the attribute $a_{i}$ has a value of $b_{k}$ is $p\left(b_{k}\right)=n_{b_{k}} / n$, where $n_{b_{k}}$ represents the number of times that the commodity consumed by the user has a value of $b_{k}$ on the attribute $a_{i}$ or is located in the interval $b_{k}$.

(iii) Step 3: calculate attribute weight through information entropy. The information is $H\left(a_{i}\right)=-\sum_{b_{k} \in v_{i}} p\left(b_{k}\right) \log p\left(b_{k}\right)$ of the attribute $a_{i}$, the information entropy is normalized $H^{\prime}\left(a_{i}\right)=H\left(a_{i}\right) / \sum_{i=1}^{m} H\left(a_{i}\right)$, and the weight of the attribute $a_{i}$ is $W_{t}=1-H^{\prime}\left(a_{i}\right)$.

(iv) Step 4: adjust the attribute weight according to the change frequency of attribute value. The change frequency of attribute value $v_{i}$ is $q_{i}=n^{*} / n-1(n \geq 2)$, where $n *$ is the number of times the values of adjacent attributes actually change. The weight of adjustment attribute is $w_{i}=w_{i} \times\left(1-\beta \times q_{i}\right), \beta$ is the adjustment range parameter, and the larger $\beta$ is, the larger the adjustment range is.

(v) Step 5: normalization of attribute weights: the normalization formula is $W_{t}^{\prime \prime}=W_{i}^{\prime} / \sum_{i=1}^{m} W^{\prime}$.

(vi) Step 6: attribute value preference calculation: the user's score is based on ten-point system, and the mapping relationship between score $\theta$ and score coefficient $\eta$ is $=\xi=\theta-\delta / 10-\delta$, which is the lowest score of all goods consumed by users. The numerical attribute value preference is adjusted by forgetting factor, the calculation formula of forgetting factor is $\lambda=\xi \times p\left(v_{i}^{\text {new }}\right), v_{i}^{\text {new }}$ is the value of the latest consumer goods on attribute $a_{i}, p\left(v_{i}^{\text {new }}\right)$ is the probability of attribute $a_{i}$ taking $v_{i}^{\text {new }}$, and the adjustment formula is $V_{i}^{\prime}=\lambda v_{i}^{\text {new }}+(1-\lambda) V_{i}$. There are at most $i$ elements stored in the preference set of nonnumerical attribute values, and the preference attribute values are updated 
according to the first-in-first-out principle, and the size of $i$ is the empirical value 5 .

$\begin{aligned} & \text { Output is user's new consumption } \\ & \text { preference }\left\langle a_{i}, v_{i}^{\prime}, w_{i}^{\prime \prime}\right\rangle \text {. }\end{aligned}$

The recommendation algorithm based on consumer preference calculates the similarity between consumer preference $\left\langle a_{i}, v_{i}^{\prime}, w_{i}^{\prime \prime}\right\rangle$ and commodity $\left\langle d_{i}, g_{i}\right\rangle$, matches the consumer preference and commodity, generates a recommendation list according to the matching degree, and recommends the top- $N$ commodities to users in turn. The recommended process is as follows:

(i) Step 1: obtain the attributes, attribute values, and attribute weights of $T$-type commodities from the current user consumption preference model $U_{t}$ and commodity model $R_{t}$, respectively.

(ii) Step 2: if the value of the attribute $a_{i}$ is numerical data, the similarity between the user's consumption preference and the commodity on the $i$ th attribute is $S_{i}=\left|v_{i}-g_{i}\right| / \max \left\{v_{i}, g_{i}\right\}$. If the value of the attribute $a_{i}$ is nonnumerical data, the similarity between the user's consumption preference and the commodity on the $i$ th attribute is $S_{i}=v_{i} \cap g_{i} / v_{i} \cup g_{i}$. The similarity between consumer preference and commodity $\operatorname{issim}\left(U_{t}, R t\right)=\sum_{i=1}^{m} w_{i} \times s_{i}$.

(iii) Step 3: sort the goods from high to low according to the similarity, and return the top- $N$ goods list to the user.

2.3. Traditional Association Rule Recommendation Algorithm. Because the sites in the field of e-commerce are built based on the Internet and the convenience of using the Internet to obtain information, each site can provide very personalized, transparent, and humanized recommendations. In recent years, knowledge discovery has been paid more and more attention in the field of artificial intelligence, and an important research direction of knowledge discovery is the mining of association rules. Association rules are also known as "shopping basket" analysis, because its main research object is e-commerce order database, and its main purpose is to find some association and combination relationships between transaction items from the order database. Apriori algorithm is the most commonly used core algorithm in association rules mining. Its core idea is to find the elements whose frequent item sets are $K$ first and then find the elements whose frequent item sets are $K+1$ according to $K$ frequent item sets. It is mainly divided into two steps:

(1) enerating all $K$-frequent item sets, firstly scanning the transaction database to obtain an item set data, calculating the support degree of an item set (i.e., the number of times the data item set $X$ appears in the transaction database), eliminating the result that the support degree is less than the preset minimum support threshold, and retaining an item frequent set. Then, binomial set is constructed; that is, the results of a frequent set are combined in pairs, and its support degree is calculated, and the item set whose support degree is not less than the preset minimum support degree is reserved. Similarly, $K$-frequent item sets can be generated by mining.

(2) The second is generating trusted association rules from $K$-frequent item sets; the confidence level (the ratio of the number of transactions containing both $X$ and $Y$ in the transaction database $D$ to the number of transactions containing $X$ in $D$ ) is calculated for the frequent item sets obtained by mining. The minimum confidence threshold is the boundary of whether the association rules generated are trusted association rules, and the value of confidence level indicates the degree to which the association rules can be trusted.

From the above analysis, it can be seen that Apriori algorithm has always been searching for frequent item sets in iteration, so the biggest problem is to scan the database many times and produce a large number of quasifrequent item sets or candidate sets, which is inefficient and low in performance, especially when facing massive data. Of course, since Agrawal first proposed association rules, many experts and scholars in the field of data mining at home and abroad have made in-depth research on association rule mining methods and proposed many algorithms. Most of them are improved and extended based on Apriori algorithm.

(1) By scanning the transaction database DB for the first time, the set $F$ of frequent items and the support degree of each frequent item are obtained, $F$ is sorted in descending order of support degree, the result $L$ is obtained, and the Header Table is constructed accordingly.

(2) Scan the transaction database DB for the second time. After removing infrequent items from each read transaction, press $L$ in the first step. After sorting, create a path of FP tree with null as the root node, and add 1 to the count of items on the path. In the process of inserting FP tree, find the corresponding items in the header table and establish a pointer index. And so on, continue to insert other transactions until their complete FP tree is built.

As can be seen from the above steps, FP-Growth algorithm only needs to scan the database twice. Compared with Apriori algorithm, FP-Growth algorithm greatly reduces traversal times, which will greatly improve the efficiency of the algorithm. In addition, FP-Growth algorithm does not need to generate candidate sets like Apriori algorithm, but it adopts divide-and-conquer strategy, which compresses the database providing frequent item sets into an FP tree and generates association rules, which also greatly improves the efficiency of the algorithm.

\section{Data Analysis of Tourism Enterprise Value Platform}

3.1. Travel Analysis of Tourism Platform. Taking May 1, 2021, as the research time point, this paper analyzes the correlation between the flow of people and tourism income from the number of people on May 1 in different periods, as shown in Figure 1. 


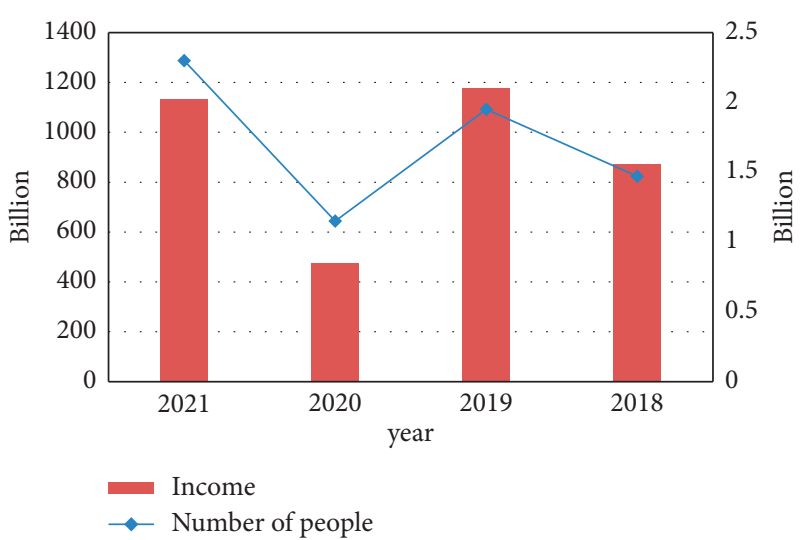

FIgURE 1: Correlation between tourism income and number of tourists.

It can be seen from Figure 1 that there is a positive correlation between tourism income and population. Due to the serious epidemic situation in 2020 , the number of tourists is relatively small, the number of tourists in the region increases, and the cost is relatively small. In 2019 and 2018, the relationship between the number of tourists and income and expenses is more obvious, mainly due to the large number of people traveling out of the province and the large cost. In 2020, the number of people is relatively small, mainly due to the epidemic situation. Many scenic spots and tourist areas implement strict policies, resulting in a small number of people and less tourism revenue. In 2021, only when the epidemic situation is well controlled will the number of tourists increase obviously and the income increase.

However, under different platforms, mobile APP is more used to realize travel reservation and travel strategy, and different regions and times can realize relative APP travel services such as strategy information, travel reservation, hotel accommodation, transportation, food, and beverage. Figure 2 shows the number of mobile APP visits from June 2019 to December 2020.

It can be seen from Figure 2 that, due to the epidemic situation, the number of people visiting APP in February 2020 was small, mainly because the national tourism was suspended and the number of visits was relatively small. With the effective control of the epidemic situation, the number of tourists nearby gradually appeared, and the number of people visiting the travel APP also increased from March to December. However, compared with the number of visits before January 2020, the minimum number of visits before the epidemic is equivalent to the maximum number of visits after the epidemic.

Nowadays, users have already adapted to the whole process of corresponding tourism projects by using the network, and tourism users have become accustomed to preparing trips and spending and sharing in advance on the travel app. This also indicates that the urgent need of the tourism mobile market has always existed. Judging from the number of newly added tourism apps every day, the tourism mobile applications in the market have maintained steady growth. Take April 2021 as an example to illustrate the increase of APP in Figure 3.
The increase of APP data in April showed irregularity, but the number of weekends such as April 3rd, April 4th, April 10th, and April 11th decreased obviously. Overall, the highest points have the largest number on Wednesdays and Thursdays.

In the tourism industry, the functions that can be realized on the APP are mainly as follows: hotel accommodation, ticketing platform, comprehensive tourism services and travel, etc. The distribution ratio of the above functions in the vertical APP is shown in Figure 4.

The choice of travel mode under various tourism platforms is also an important link in tourism. In the mainstream mode of travel, people use APP to choose the number of visits in different time periods, as shown in Figure 5.

As can be seen from Figure 5, the number of visits to the platform increased significantly in August, September, and October 2020. In these three months, it shows that the epidemic situation is well controlled, and people have more holidays, especially in October, with the largest increase. It shows that there are more people who have tourism plans in the three months of a year; so long as the tourism routes and reasonable tourism plans are arranged reasonably, tourism income can be provided.

Nearly $80 \%$ of tourism consumers use tourism-related apps to arrange trips, including information inquiry, accommodation, and delivery.

It is used most in the three links of reservation. Nowadays, tourism consumers have become accustomed to preparing their journeys in advance on the travel App, and the travel App has been able to provide travel services in the whole process chain, which greatly enhances users' willingness to download and use. The specific scale is shown in Figure 6.

3.2. Market Application Analysis of Tourism Value Platform. This section analyzes the resources under the tourism value platform from the aspects of strategy information, travel reservation, hotel accommodation, transportation, and food and beverage. From the perspective of various domestic tourism platforms, this paper mainly analyzes the main application scenarios of tourism platforms in these aspects, and the specific tourism enterprises are shown in Table 2.

The following form the various functions to analyze the application of the tourism platform, for the tourism platform access data analysis. Through relative data, this paper analyzes the tourism distribution under different platforms and explains five functions: strategy information, travel reservation, hotel accommodation, transportation, and food and beverage.

3.2.1. Attractions Ticketing. In the Raiders information, it mainly focuses on the platforms of Xiaohongshu, Qunar, $\mathrm{Ma}$ Honeycomb, and Public Comment. The specific data distribution is shown in Figure 7.

In the past, where the mainstream travel strategy platform went, Ma Honeycomb was no longer brilliant. Although there was still a steady increase in downloads every month, it was no longer the first echelon of travel strategy app. 


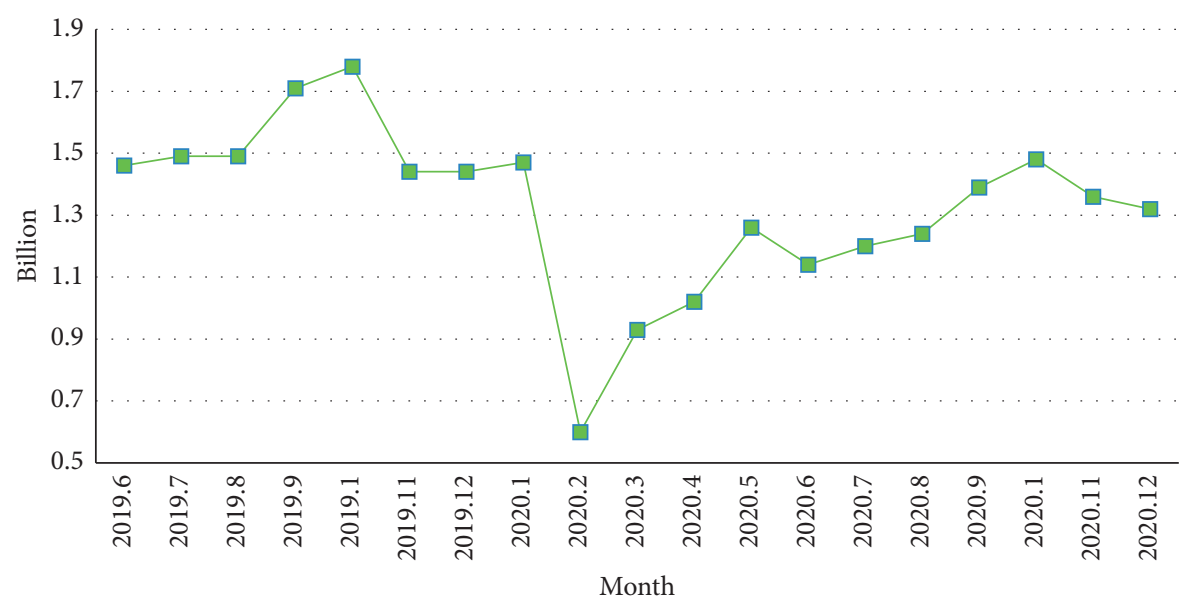

FIgUre 2: Number of apps visited in April.

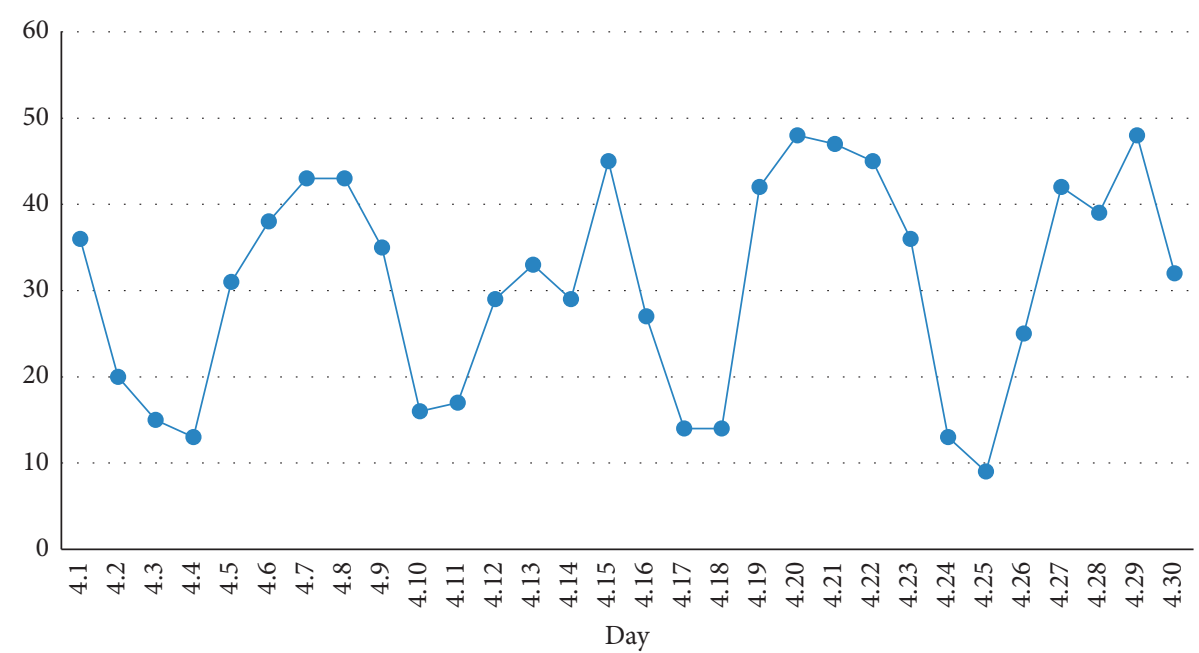

FIgure 3: Daily APP increase in April.

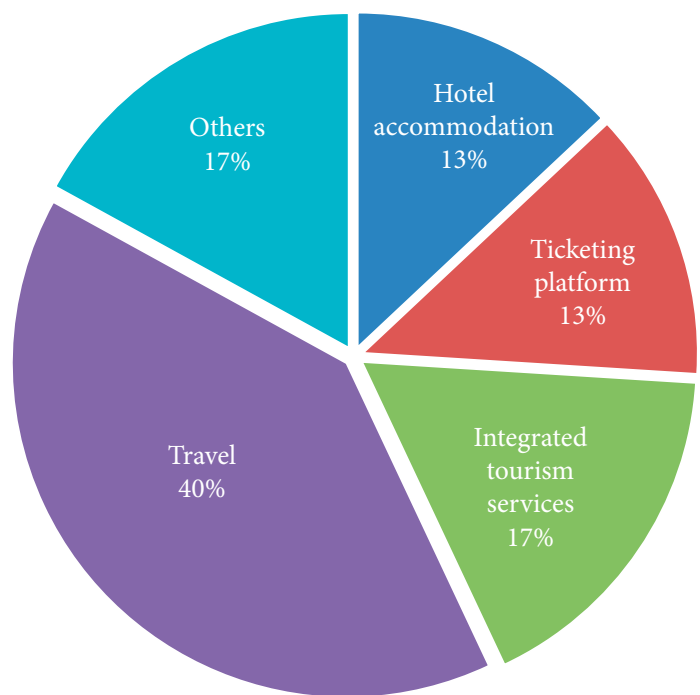

FIGURE 4: Function comparison relationship of tourism platform.
3.2.2. Scheduling. In the travel reservation information, the analysis mainly focuses on the platforms of Qunar, Same Trip, Ctrip Travel, and Flying Pig Travel. The specific data distribution is shown in Figure 8.

3.2.3. Hotel Accommodation. In the hotel accommodation information, the analysis is mainly concentrated on the platforms of Same Trip, Ctrip Travel, Flying Pig Travel, China Living Club, Airbnb, Bird Famous Residence, and Booking. The specific data distribution is shown in Figure 9.

3.2.4. Traffic Reservation. In the traffic travel information, the analysis mainly focuses on the platforms of Where to Travel, DiDi Travel, Hello Travel, and Hua Xiaozhu Taxi, among which Hua Xiaozhu Taxi is a rising star in 2020, and the specific data distribution is shown in Figure 10. 


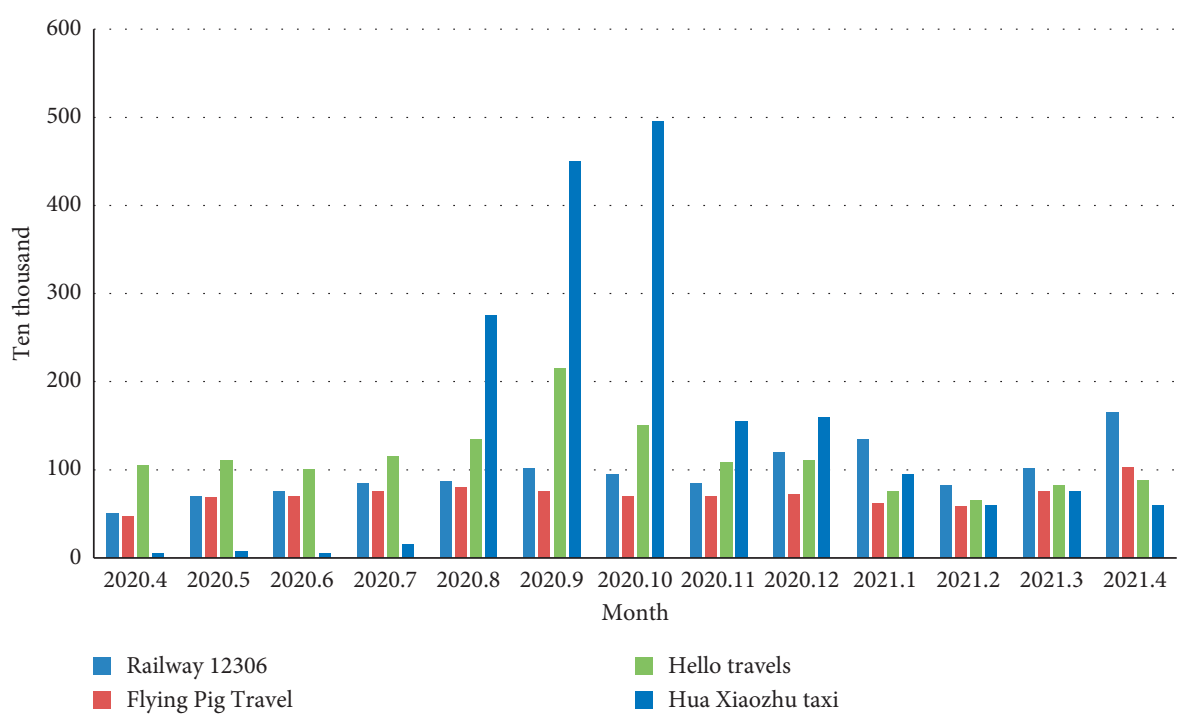

FIgURE 5: Travel visits of tourism platform.

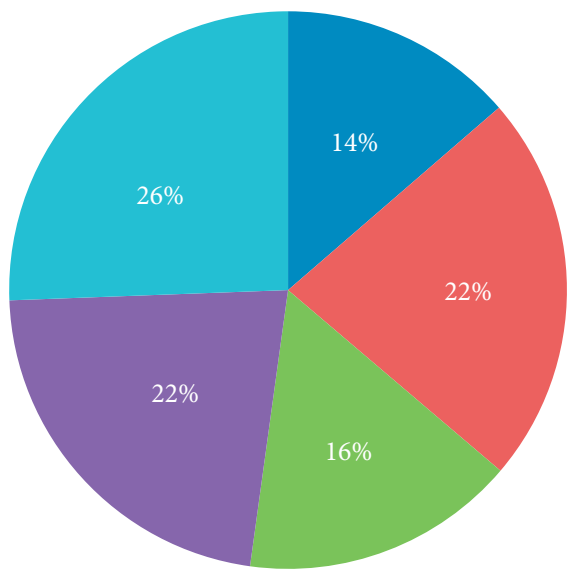

- Attractions ticketing

- Accommodation

- Equipment purchase

- Traffic reservation

- Raiders information

FIGURE 6: Utilization rate of tourism platform.

TABLE 2: Application of main functions of tourism platform.

Tourism project

Attractions ticketing

Scheduling

Hotel accommodation

Traffic reservation

Raiders information

Under the tourism platform, passengers analyze road conditions through navigation, which can effectively improve the ability of accurate location. In Gaode Map, Baidu Maps, and Tencent Maps, the growth rate of Gaode Map is much higher than the latter two from August 2020, and the specific distribution is shown in Figure 11.
Tourism enterprise

Ctrip, Where to travel, Hornet's Nest, Public Comment, Little Red Book Ctrip, Where To Travel, Same Journey, Flying Pig Travel Flying Pig Travel, China Residential Association, Airbnb Drip Travel, Flower Piglet, Harrow Trip, Tick-Tock Trip Meituan, Are You Hungry, Public Comment

3.2.5. Raiders Information. In the food and beverage information, the analysis is mainly concentrated on Meituan, ELEME, Public Comment, and Baidu Glutinous Rice. The specific data distribution is shown in Figure 12.

The catering platform with take-out function occupies an important position in the tourism catering market. 


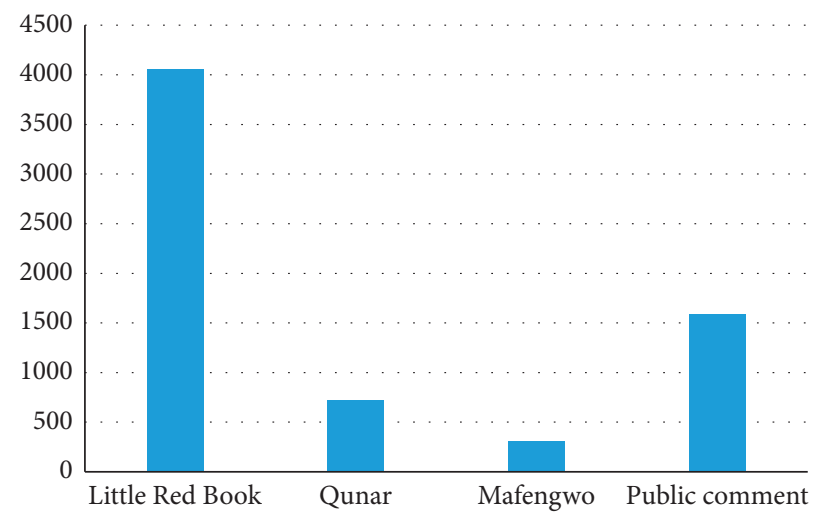

FIGURE 7: Raiders' information distribution information of tourism platform.

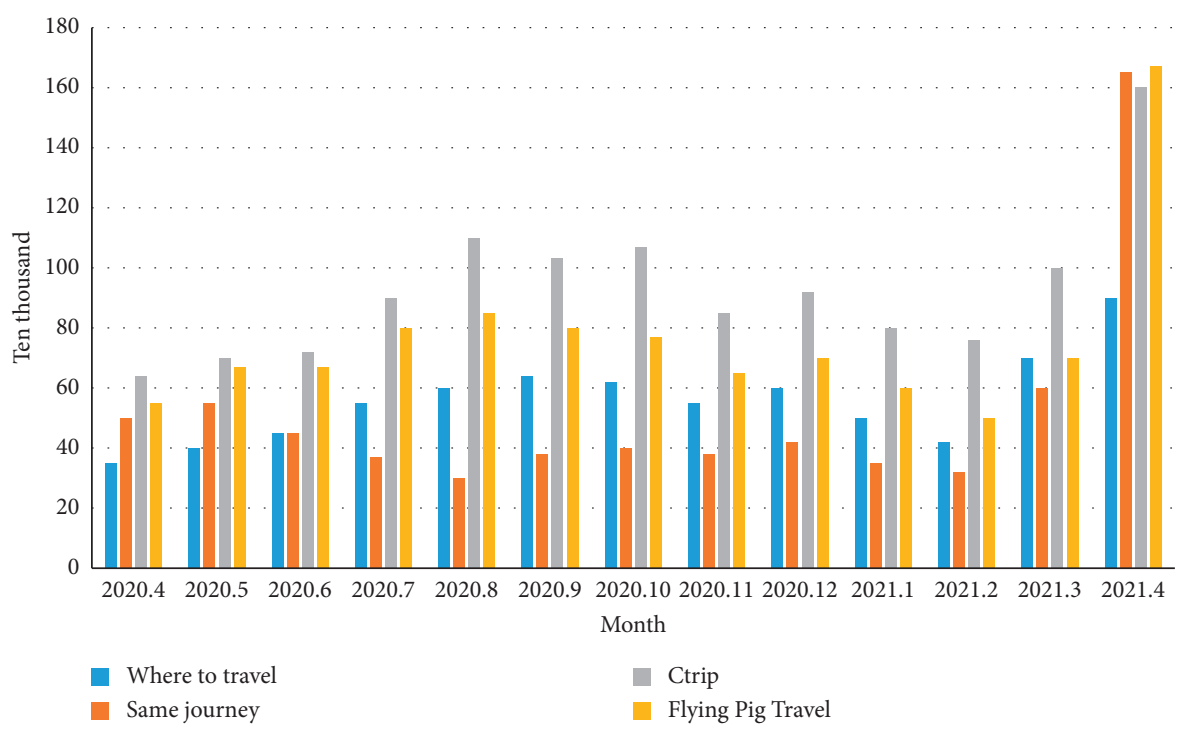

Figure 8: Travel platform access information for travel reservation.

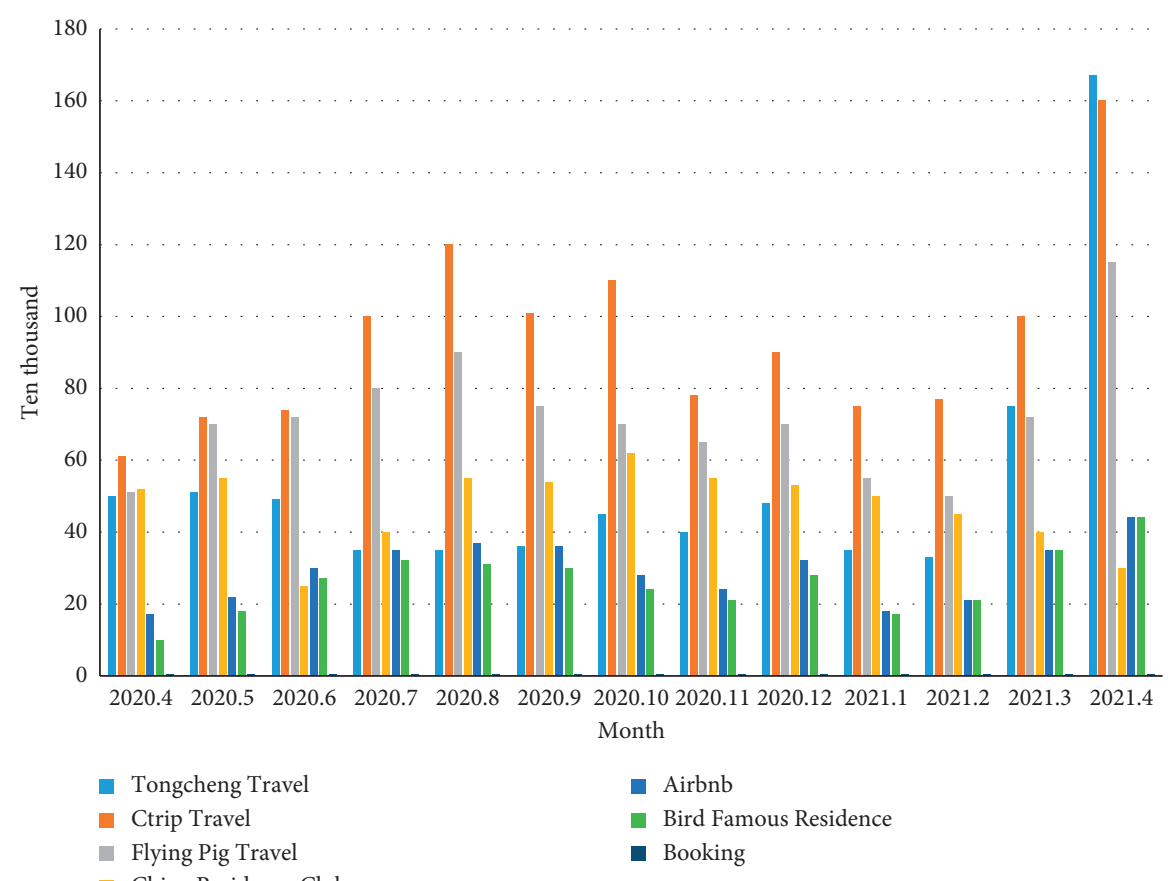

China Residence Club

Figure 9: Travel platform access information for hotel accommodation. 


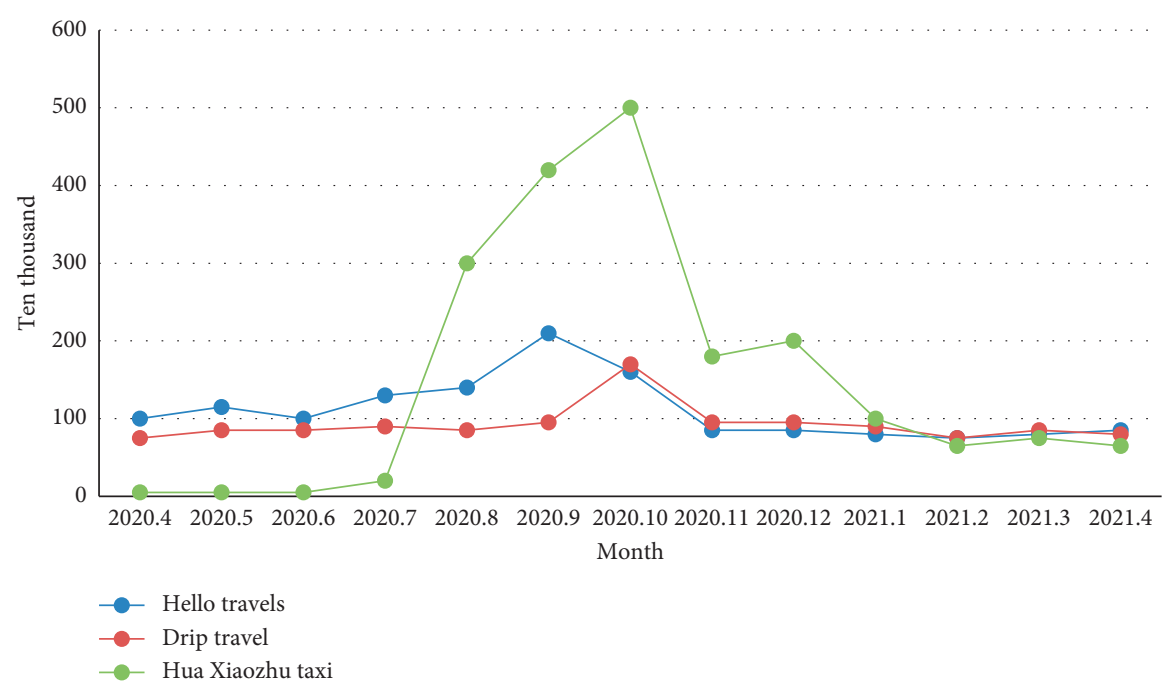

FIGURE 10: Platform information distribution of travel.

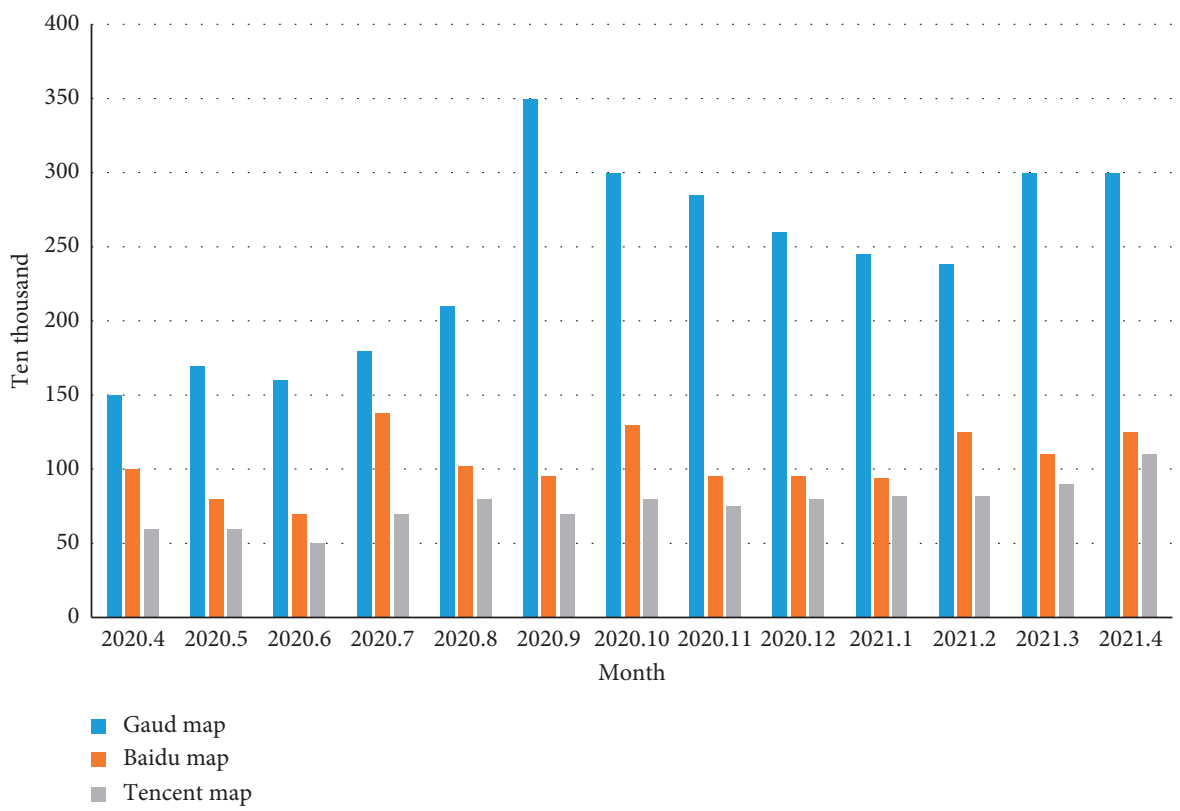

Figure 11: Travel platform distribution information of itinerary navigation.

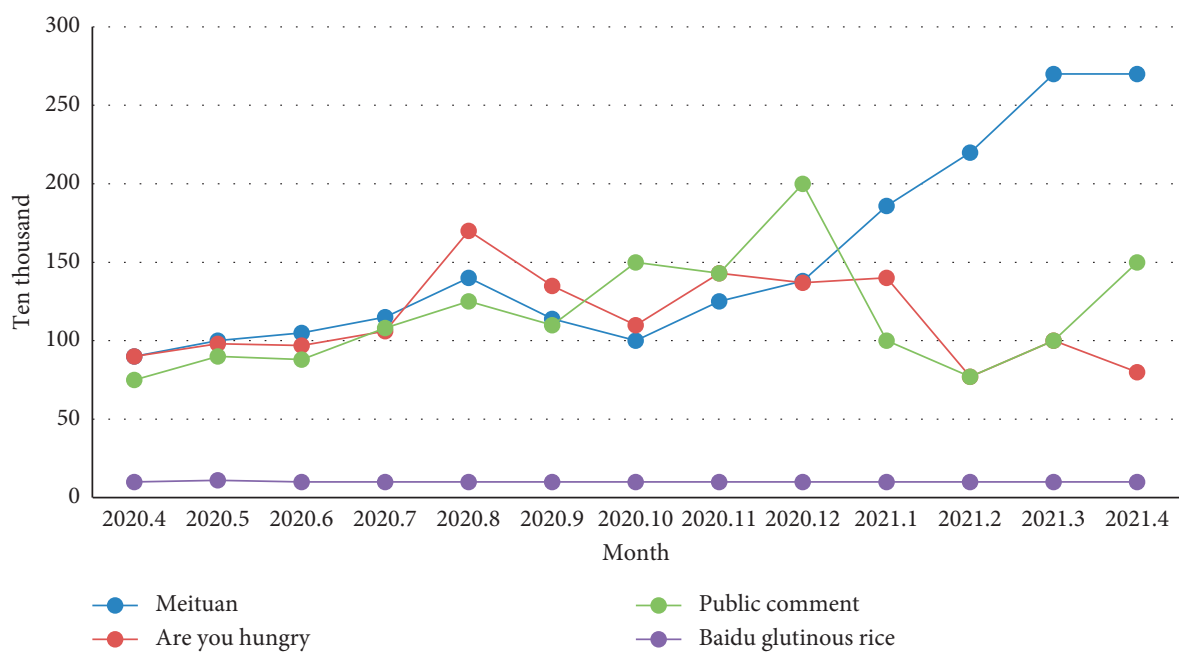

Figure 12: Distribution information of tourism platform for catering and gourmet food. 


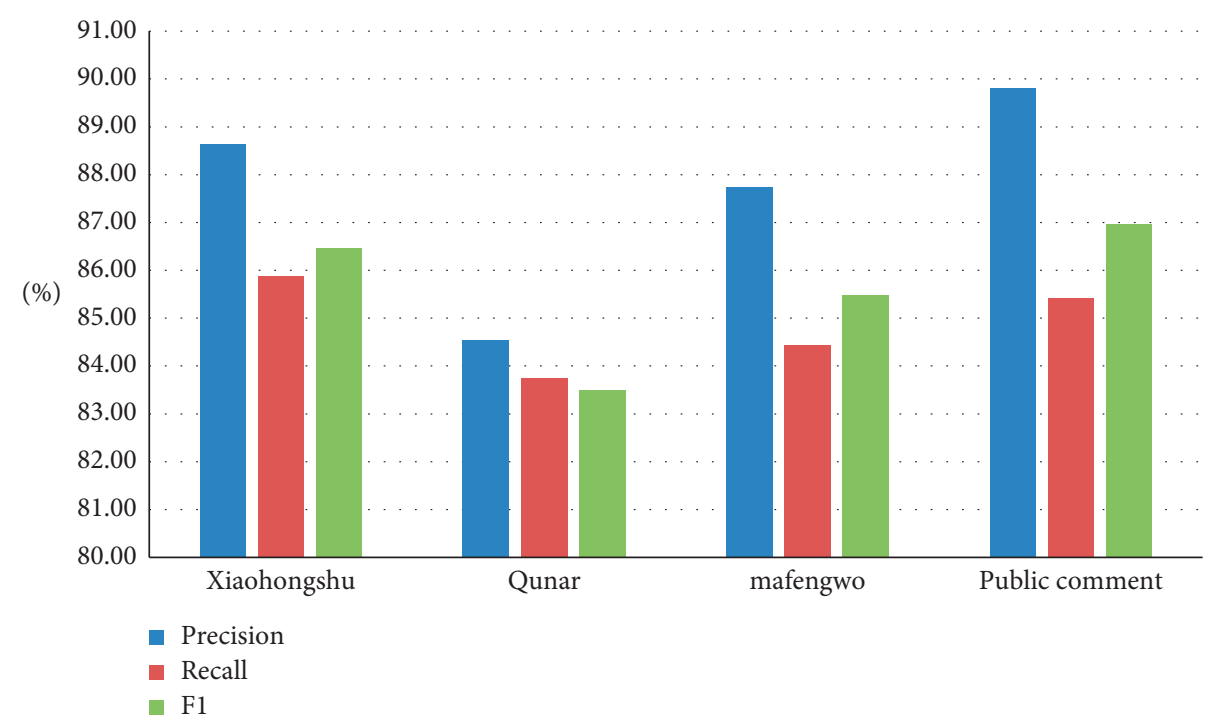

FIgURE 13: Comparison of Raiders' information recommendation performance of tourism platform.

Represented by Meituan Takeaway and Hungry Noodles, from the performance of downloads in the past year, Meituan Takeaway has a strong momentum, and since 2021, it has gradually cast off Hungry Noodles as a direct competitor. The Public Comment, which integrates comment, group purchase, and preferential payment, performed stably. The recommendation of "foodies" provides a credible reference for new visitors, which also makes Public Comment have strong vitality in the domestic tourism market all the year round. The development momentum of gourmet group buying applications is weak. Baidu Glutinous Rice and other platforms focusing on food group buying have weak development momentum, so perhaps focusing on a single group buying field is not the most suitable choice at present.

\section{Research on Recommendation Business Model under Multiplatform from the Perspective of Value Cocreation}

In the second section, three recommendation methods are introduced. Because of the differences in usage between recommendation methods, enterprises under many tourism platforms have effective analysis on different recommendation methods under various platforms. In order to maximize the operating benefits of the business model, maximizing the commercial value based on multiple platforms is the goal pursued, and achieving good user experience and sharing good tourism can effectively improve the commercial value of the tourism platform.

The following studies the business models of the above problems from different recommendation methods combined with various tourism platforms and analyzes the business models of recommendation algorithms such as collaborative filtering, association rules, and content filtering under different platforms, so as to realize the selection of the best tourism business platform and obtain the maximum benefits and results.

In order to reflect the actual test effect of the above indicators, the mean absolute error (MAE) can be used to express the score prediction accuracy, which has the advantages of simplicity and easy understanding. The MAE calculation formula is shown as follows:

$$
\text { MAE }=\frac{\sum_{u, i \in T}\left|r_{u i}-\widehat{r}_{u i}\right|}{|T|},
$$

where $r_{u i}$ indicates the actual score of the user $U$ on the commercial service $I$ provided by the travel platform and $\widehat{r}_{u i}$ indicates that the recommendation system predicts the score of the commercial service I provided by the user $U$ on the travel platform. In this paper, the difference of MAE overall error evaluation is not obvious, so this index will not be discussed.

The prediction accuracy of tourism business platform recommendation can accurately predict the user's definite preference for recommended tourism platform, which is expressed by two parameters: accuracy and recall.

$$
\begin{aligned}
\text { Precision } & =\frac{\sum_{u \in U}|R(u) \cap T(u)|}{\sum_{u \in U}|R(u)|}, \\
\text { Recall } & =\frac{\sum_{u \in U}|R(u) \cap T(u)|}{\sum_{u \in U}|T(u)|}, \\
F_{1} & =\frac{2 * \text { Precision } * \text { Recall }}{\text { Precision }+ \text { Recall }} .
\end{aligned}
$$

Through the above indicators to analyze the recommendation effect under various tourism platforms, the recommendation of each platform business is still evaluated by five main modules: strategy information, travel reservation, hotel accommodation, transportation, and food and beverage under the tourism platform. 


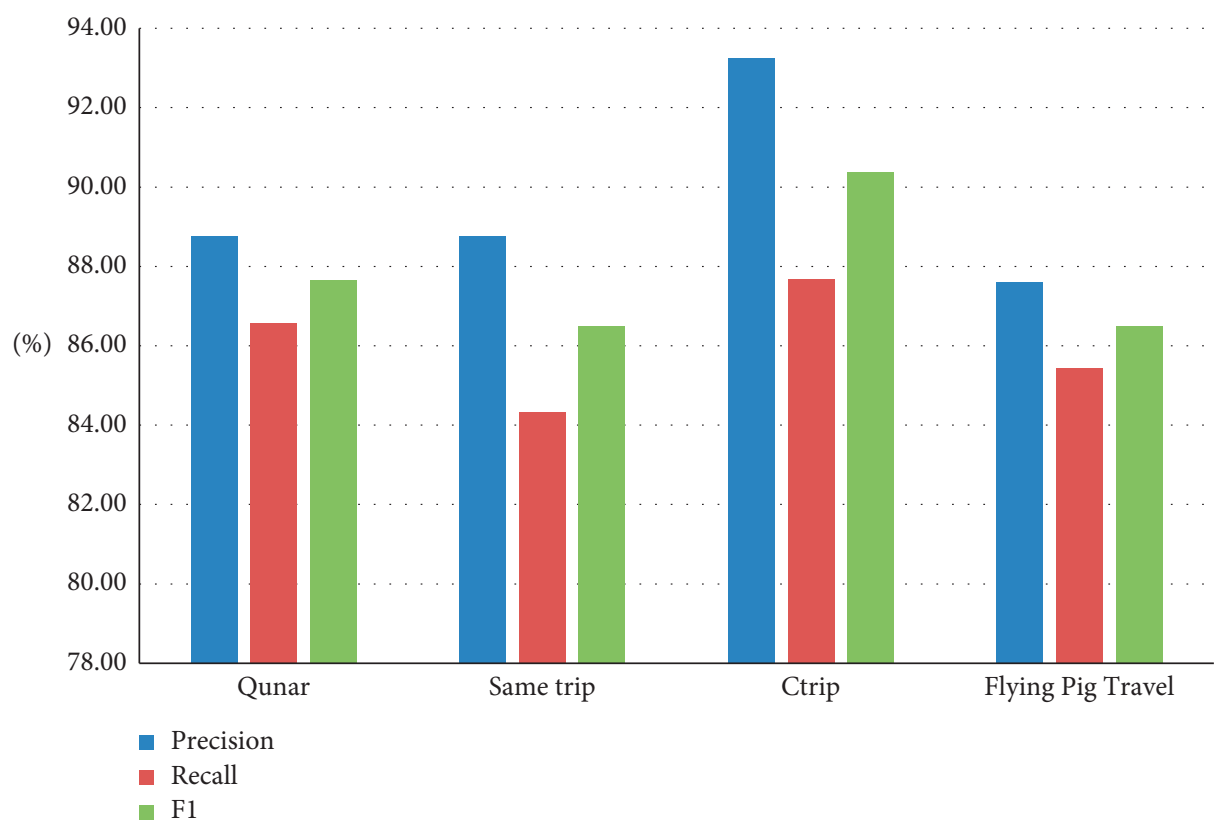

FIGURE 14: Comparison of travel booking recommendation performance of travel platform.

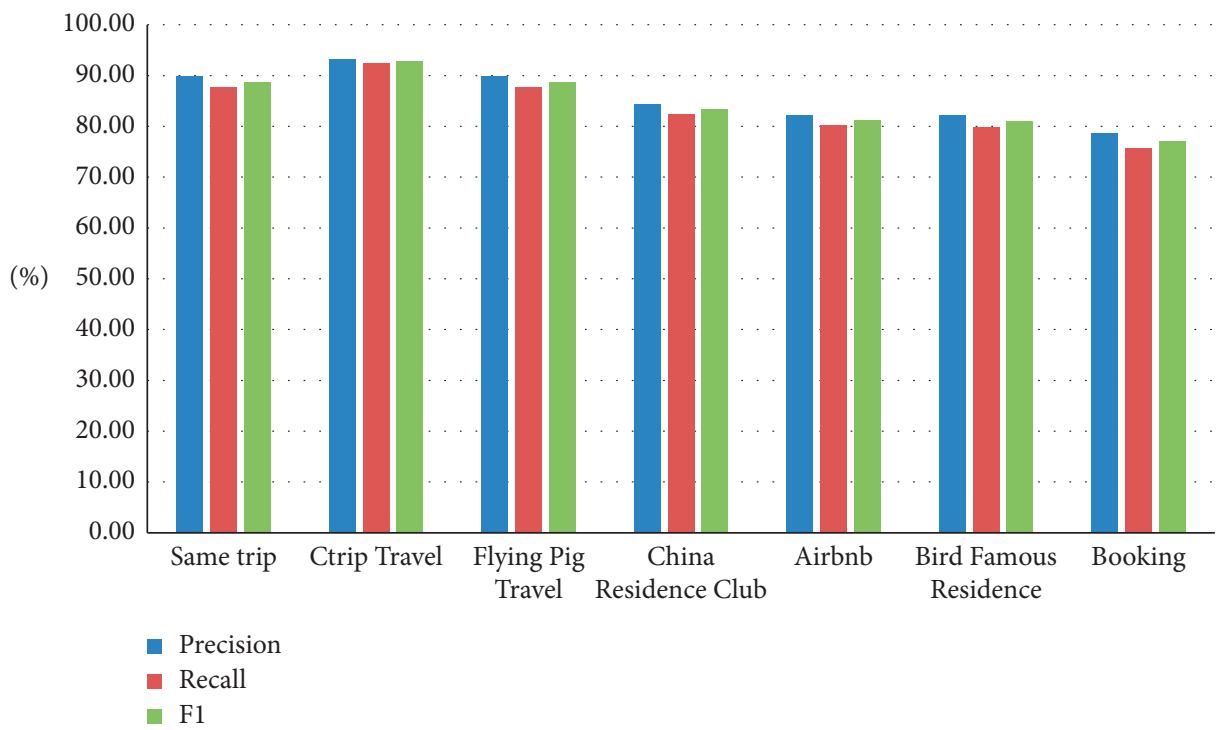

FIGURE 15: Comparison of hotel accommodation recommendation performance of tourism platform.

4.1. Attractions Ticketing. In the strategy information of tourism platform, the predetermined information of Xiaohongshu, Qunar, Ma Honeycomb, and Public Comment is mainly concentrated as the research target, and data collection and sharing are adopted. The specific data distribution is shown in Figure 13.

4.2. Scheduling. In the travel reservation information, the analysis mainly focuses on the platforms of Where to Travel, Same Trip, Ctrip Travel, and Flying Pig Travel and analyzes the accuracy rate, recall rate, and $F 1$ under various recommendation algorithms. The specific data distribution is shown in Figure 14.
4.3. Hotel Accommodation. In the hotel accommodation information, the accuracy rate, recall rate, and $F_{1}$ are mainly analyzed under several recommendation algorithms, such as Same Trip, Ctrip Travel, Flying Pig Travel, China Living Club, Airbnb, Bird Famous Residence, and Booking, and the specific data distribution is shown in Figure 15.

4.4. Traffic Reservation. In the traffic travel information, it mainly focuses on DiDi Travel, Hello Travel, Hua Xiaozhu Taxi, and so on. Road condition analysis through navigation can effectively improve the ability of accurate location. Gaode Map, Baidu Maps, and Tencent Maps are mainly used for navigation path. Accuracy rate, recall rate, and $F 1$ are analyzed on several platforms under various recommendation algorithms, among 


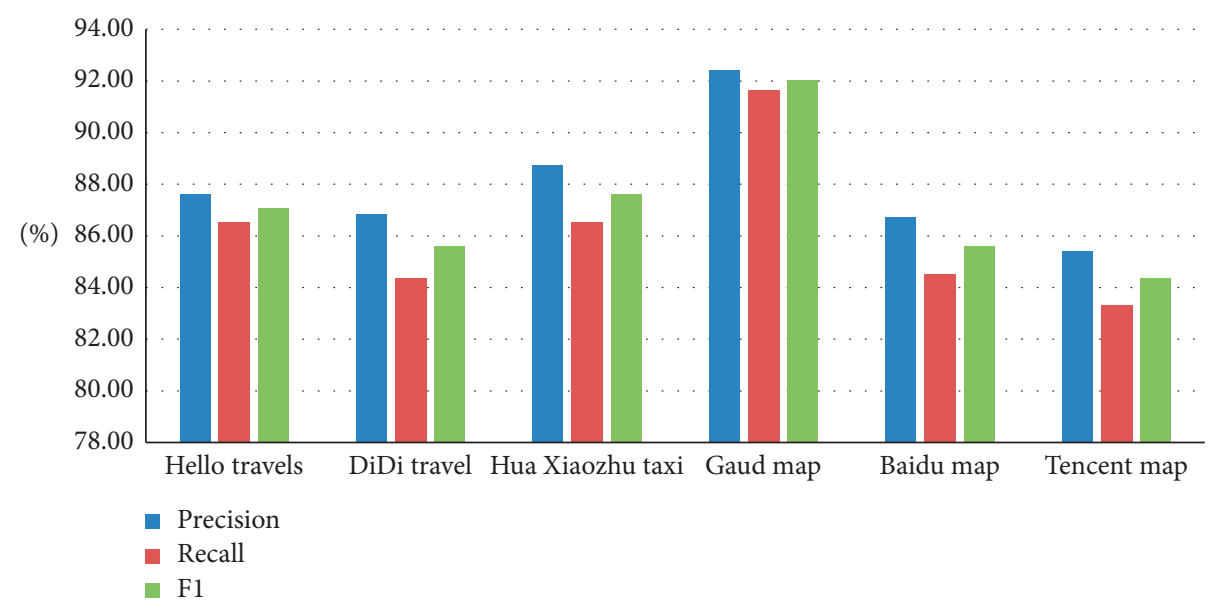

FIGURE 16: Comparison of transportation recommendation performance of tourism platform.

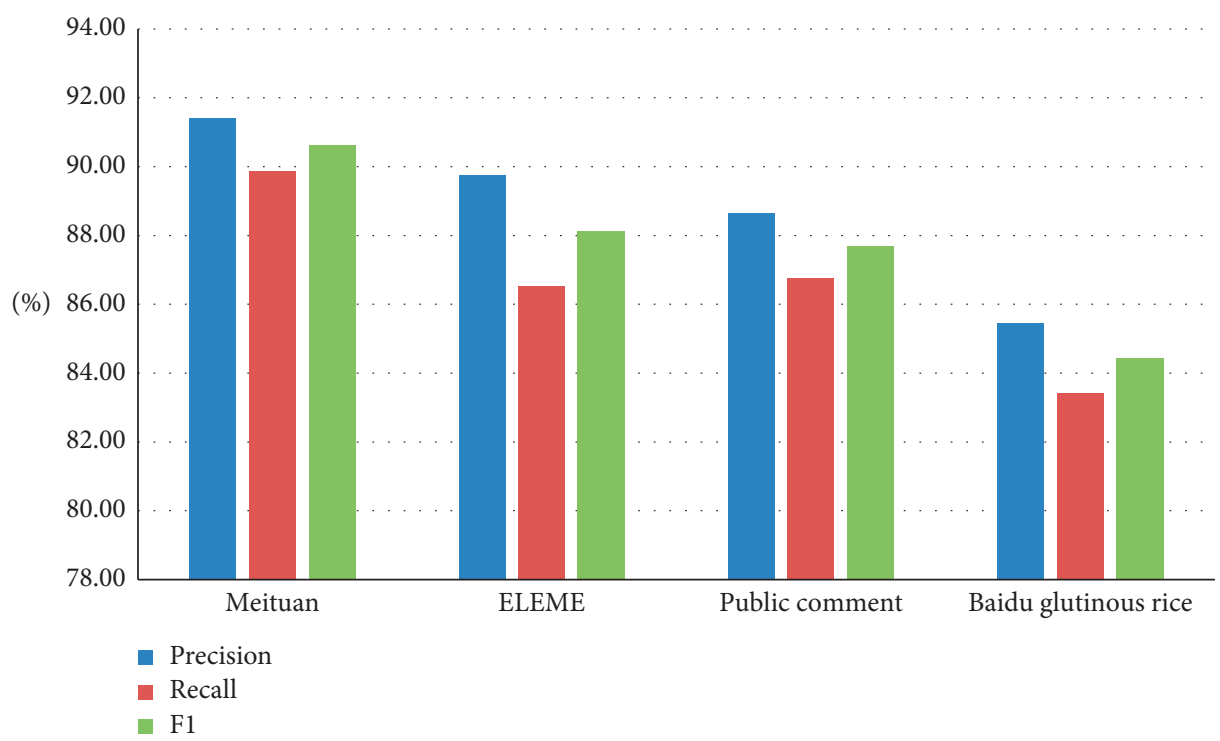

Figure 17: Comparison of food and beverage recommendation performance of tourism platform.

which Hua Xiaozhu Taxi's performance is also very good, and the specific data distribution is shown in Figure 16.

4.5. Raiders' Information. In the food and beverage information, it mainly focuses on Meituan, Hungry, Public Comment, and Baidu Glutinous Rice. The accuracy rate, recall rate, and $F 1$ are analyzed on several platforms under various recommendation algorithms. The specific data distribution is shown in Figure 17.

Analysis of the performance of the abovementioned modules, generally in the accuracy and recall rate of more than $88 \%$, all show good performance. These business models can provide better services and get better attention.

\section{Conclusion}

Considering the commercial value, the service value platform provided by tourism enterprises provides customers with high-quality services and gains the satisfaction of users in order to realize commercial value. The three service recommendation algorithms proposed in this paper are applied to the business service recommendation of tourism platform. Different recommendation algorithms have different requirements, and the optimal recommendation method is used to realize recommendation under different platforms. From the recommendation effect, the overall optimal recommendation effect is over $88 \%$, and the service requests and recommendations put forward by customers can achieve the purpose of commercial services. The future research work should analyze the difference of multiplatform recommendation, make the best recommendation method according to the customer's request, and then provide value-added services to meet the customer's needs and maximize the commercial benefits.

\section{Data Availability}

The data used to support the findings of the study are available from the corresponding author upon request. 


\section{Conflicts of Interest}

The authors declare that they have no conflicts of interest regarding this work.

\section{References}

[1] K. Meehan, T. Lunney, K. Curran et al., "Context-aware intelligent recommendation system for tourism," in Proceedings of the IEEE International Conference on Pervasive Computing \& Communications Workshops, pp. 328-331, IEEE, San Diego, CA, USA, March 2013.

[2] R. Bin, "Research on tourism service intelligent recommendation system based on apriori-MD algorithm," Applied Mechanics and Materials, vol. 651-653, pp. 1642-1646, 2014.

[3] H. Li, S. Zhang, J. Shi et al., "Research and design of intelligent learning system based on recommendation technology," Control \& Intelligent Systems, vol. 47, no. 1, pp. 43-49, 2019.

[4] O. Boulaalam, B. Aghoutane, D. El Ouadghiri et al., "Proposal of a big data system based on the recommendation and profiling techniques for an intelligent management of Moroccan tourism - ScienceDirect," Procedia Computer Science, vol. 134, pp. 346-351, 2018.

[5] T. Wang, "The application of ontology technology in E-commerce recommendation system," Advances in Intelligent and Soft Computing, vol. 156, pp. 71-76, 2012.

[6] H. Teke, P. Borle, M. Tiwari et al., "An intelligent recommendation system to enhance the travel services," International Journal of Advance Research and Innovative Ideas in Education, vol. 1, no. 4, pp. 2395-4396, 2015.

[7] G. Biiyuközkan and B. Ergün, "Intelligent system applications in electronic tourism," Expert Systems with Applications, vol. 38, no. 6, pp. 6586-6598, 2011.

[8] A. Smirnov, A. Kashevnik, A. Ponomarev et al., "Proactive recommendation system for $\mathrm{m}$-tourism application," in Proceedings of the International Conference on Business Informatics Research, September 2014.

[9] F. Ricci and H. Werthner, "Case base querying for travel planning recommendation," Information Technology \& Tourism, vol. 4, no. 3-4, pp. 215-226, 2001.

[10] K. Meehan, T. Lunney, K. Curran et al., "Aggregating social media data with temporal and environmental context for recommendation in a mobile tour guide system," Journal of Hospitality and Tourism Technology, vol. 7, no. 3, pp. 281-299, 2016.

[11] G. Tong, B. Guo, J. Zhang et al., "Crowd travel: leveraging heterogeneous crowdsourced data for scenic spot profiling and recommendation," Pacific Rim Conference on Multimedia, vol. 9917, pp. 617-628, 2016.

[12] E. S. Khorasani and S. Meybodi, "A framework to improve E-tourism experience by using intelligent portal," in Proceedings of the Seventh International Conference on Information Technology: New Generations, April 2010.

[13] H. Fu, G. Manogaran, K. Wu et al., "Intelligent decisionmaking of online shopping behavior based on Internet of things," International Journal of Information Management, vol. 50, pp. 515-525, 2020. 